\title{
ANTIBACTERIAL ACTIVITY OF SILVER NANOPARTICLES ON FIXED RETAINER ADHESIVE TOWARD TREPONEMA DENTICOLA
}

\author{
JESSICA SANTOSO, MARIA PURBIATI*, KRISNAWATI \\ Department of Orthodontics, Faculty of Dentistry, Universitas Indonesia, Jakarta, 10430, Indonesia. Email: mariapurbiati@gmail.com
}

Received: 13 September 2018, Revised and Accepted: 10 December 2018 and 08 February 2019

\begin{abstract}
Objective: The objective of this study was to develop an antibacterial flowable composite containing silver nanoparticle (AgNP) for the prevention of periodontal disease in fixed retainers.

Methods: About 1\% AgNP was incorporated into a commercial composite (tetric flow). The experimental and control products were used to bond fixed retainers to 28 extracted mandibular first premolars. The samples were randomly divided into four groups (n=7): Premolar bonded with fiber-reinforced composites and tetric flow (F1); premolar bonded with fiber-reinforced composites and AgNP-enhanced tetric flow (F2); premolar bonded with multistranded wires (MW) and tetric flow (M1); and premolar bonded with MW and AgNP-enhanced tetric flow (M2). Each sample was submerged in a test tube containing bacterial Treponema denticola solution and was incubated for $24 \mathrm{~h}$ and $37^{\circ}$ temperature. The bacterial colony in each group was counted and analyzed.
\end{abstract}

Results: This study showed that there was significant difference of T. denticola colony count between groups with and without AgNP-enhanced composites in both types of retainers.

Conclusion: AgNP-enhanced flowable composites reduce the bacteria T. denticola colony count and possibly inhibit periodontal disease.

Keywords: Periodontal disease, Treponema denticola, Fixed retainer, Silver nanoparticle.

(C) 2019 The Authors. Published by Innovare Academic Sciences Pvt Ltd. This is an open access article under the CC BY license (http://creativecommons. org/licenses/by/4. 0/) DOI: http://dx.doi.org/10.22159/ijap.2019.v11s1.18123

\section{INTRODUCTION}

Retention is a crucial stage in orthodontics. Some factors cause unstability of tooth position after orthodontic treatment. Gingival and periodontal tissue involved in orthodontic tooth movement requires time to reorganize following active orthodontic appliances removal. Due to this surrounding soft tissues condition, tooth position after appliances debonding is still unstable. Growth is also one of the factors that influence post-orthodontic treatment tooth stability as well as chewing force. Therefore, orthodontic retainers on the retention phase control forward tooth position and occlusal relationship [1].

Two kinds of retainers are available on the market: Removable and fixed retainers. Removable retainers are suitable for patients with growth disturbance; yet, it requires the patient's cooperation. Fixed retainers are good for long-term retainers [1].

Fixed retainers are made of multistrand wire, precasted wire, and fiberreinforced composites (FRCs). Multistrand wires are flexible wires that are bonded to each tooth. The flexibility of the wire accommodates physiologic tooth movement and is very effective in preventing tooth rotation. Precasted wires are only bonded to canines and are suitable for maintaining intercanine width but cannot prevent tooth rotation. FRCs were developed as an esthetic alternative to orthodontic retainers and can be used for patients who are allergic to nickel, whereas nickel is the important component to achieve flexibility property in metal-based retainers (multistrand and precasted wire). Burstone and Kuhlberg suggested the use of FRCs as an active and passive orthodontic device [1-4].

Fixed retainers are commonly used to maintain intra-arch stability, diastema, space for pontic or implant, and post-extraction space that has been closed; then, it needs to be used for a quite long time period $[1,3,5]$. However, fixed retainers increase the area of bacterial, biofilm, plaque, and calculus accumulation that spreads gingivally producing substrate that is suitable for biofilm precipitation. Bacterial adhesion is the key to oral biofilm pathogenic formation that causes periodontal problems, gingival recession, and increased probing depth, whereas Treponema denticola, Porphyromonas gingivalis, and Bacteroides forsythus are red complex bacteria related to the development of periodontal disease. Especially, T. denticola is played an important role in periodontal diseases development, those are on the early onset, necrotizing ulcerative gingivitis, and acute periodontitis $[6,7]$.

To take the advantages of fixed retainer and to avoid periodontal problems as the side effect, it needed to develop fixed retainer with a local site antibacterial properties. This is also because the use of antibacterial gargle cannot be used every day for a long-term usage due to the balance of normal flora has to be maintained. Silver has a wide-spectrum antibacterial effect, better than the other metal-based antibacterial $[6,8,9]$. Silver nanoparticle (AgNP) is used in medical for many purposes including to coat metal surfaces. The objective of this study was to develop an antibacterial flowable composite as a fixed retainer adhesive containing AgNP for the prevention of periodontal disease in fixed retainers.

\section{METHODS}

After getting ethical approval from the Research Ethics Commission, Faculty of Dentistry Universitas Indonesia, this research study was done at the Laboratory of Oral Biology of the same faculty. This study was an in vitro laboratory experimental study.

Fixed retainer needs composite as an adhesive agent to bond the retainer to the teeth. This study used a common commercial adhesive agent that was light-cured flowable composite Tetric Flow (Ivoclar Vivadent, Schaan, Liechtenstein). The control group samples used tetric flow only, and the experimental group samples used tetric flow mixed with $20 \mu \mathrm{g}$ particle size AgNP 1\% (w/w). There were two types of fixed 
retainers included in this study: FRCs fixed retainer (everStick Ortho, GC Corp, Japan) and multistranded wires (MW) fixed retainer (Wildcat lingual retainer, Dentsply Sirona, USA).

The control and experimental material were used to bond the fixed retainers to 28 extracted mandibular first premolars. The samples were randomly divided into four groups ( $n=7$ in each group): Premolar bonded with FRC fixed retainer and tetric flow (F1); premolar bonded with FRC fixed retainer and AgNP-enhanced tetric flow (F2); premolar bonded with MW fixed retainer and tetric flow (M1); and premolar bonded with MW fixed retainer and AgNPenhanced tetric flow (M2). Each sample was submerged in a test tube containing T. denticola bacterial solution (TD ATCC 33520) composed of $3 \mathrm{ml}$ brain heart infusion (BHI) broth and $30 \mu \mathrm{L}$ TD solution. The test tubes were cultured under anaerobic conditions for $24 \mathrm{~h}$ and $37^{\circ}$ temperature.

Each sample was swabbed at the retainer composite junction using sterilized cotton buds and was cultured using BHI agar under anaerobic conditions for $24 \mathrm{~h}$, and the colony count was observed with spread plate technique and counted in colony-forming unit. Intraclass correlation (ICC) is used in testing intra- and inter-observer reliability. Data were analyzed using SPSS 20.0. Shapiro-Wilk used to test the normality of the data $(n<50)$. Analytical test used was unpaired t-test.

\section{RESULTS}

ICC tests of $10 \%$ samples showed strong (ICC value almost 1.000) intraobserver and interobserver reliability between observers. Interobserver ICC value was 0.993 , whereas intraobserver ICC value was 0.997. Shapiro-Wilk normality test significance level was at $\mathrm{p}<0.05$. According to the test, most of the data distribution was normal except the data of FRC with AgNP (Group F2) and the data of MW without AgNP (Group M1) which were giving p value under 0.05 (Table 1). For normal distribution data, the hypothetical test was using independent t-test, whereas the Groups F2 and M1 were using Mann-Whitney.

T. denticola bacterial colony count of premolars bonded with FRC retainer and AgNP-enhanced tetric flow was significantly lower than in those bonded with FRC retainer and tetric flow without AgNP. The colony count of premolars bonded with MW and AgNP-enhanced tetric flow was significantly lower than in those bonded with MW and tetric flow without AgNP (Table 2).

To show the difference of FRC and MW fixed retainers on the decrease of bacterial colony count between adhesive material with and without enhancement of AgNP, the hypothetical t-test was done. (Table 3).

The difference of the decrease of bacterial colony count on premolars bonded with FRCs retainer-AgNP-enhanced tetric flow and MW-AgNPenhanced tetric flow was not statistically significant difference.

\section{DISCUSSION}

Fixed retainers are commonly used for long-term retention. It is commonly used to conserve the position of lower incisive, postdiastema cases, maintain space for pontic or implant, and maintain post-extraction space closing [1]. However, they increase the incidence of periodontal disease by increasing the area of bacterial accumulation that spreads gingivally producing substrate that is suitable for biofilm precipitation $[1,3,6,7,10]$. Periodontal diseases are complex multifactorial diseases caused by polymicrobial subgingival biofilm with immune and inflammatory responses. The bacterial species P. gingivalis, T. denticola, and Tannerella forsythia are strongly implicated in the development of periodontal disease and together as the "red complex" [11]. T. denticola is obligate anaerobic, Gram-negative, motile, and highly proteolytic spirochete bacterium. It is predominantly subgingival oral spirochete closely associated with periodontal disease. It has extracellular proteolytic activity, anaerobic amino
Table 1: Data distribution normality test of Treponema denticola colony count of each group

\begin{tabular}{ll}
\hline Fixed Retainer+Adhesive & p value \\
\hline FRC without AgNP (F1) & 0.752 \\
FRC with AgNP (F2) & $0.028^{*}$ \\
MW without AgNP (M1) & $0.039^{*}$ \\
MW with AgNP (M2) & 0.389 \\
$\Delta$ FRC & 0.771 \\
$\Delta$ MW & 0.057 \\
\hline
\end{tabular}

${ }^{*} \mathrm{p}<0.05$. FRC: Fiber-reinforced composite, AgNP: Silver nanoparticle, MW: Multistranded wires

acid fermentation, toxic metabolic production, and outer membrane vesicles [12,13]. T. denticola possesses several virulence factors such as the major surface protein (MSP), cell-associated lipooligosaccharide, chymotrypsin-like protease (dentisilin), peptidoglycan, cystalysin, several peptidases, and a phosphatase which causes host immune cells to express molecular mediators that destroy periodontal connective tissue [14]. T. denticola causes a cytopathic effect on fibroblast cultures by inhibiting proliferation, cytoskeletal remodeling that can cause cell breakdown, and shrinkage. Bacterial adhesion on epithelial cells can cause morphological destruction $[13,15]$.

AgNP on fixed retainer adhesive prevents bacterial adhesion on the tissue surface, thus, preventing biofilm formation. This is supported by the previous study about the antibacterial properties of removable retainers with AgNPs [16].

AgNP provides a large surface area for contact with bacteria which may allow the particles to attach to the cell membrane and easily penetrate into the bacteria. It also interferes with the respiratory chain in the bacterial mitochondria, resulting in cell death [17]. AgNP causes destruction of respiratory chain, alteration of bacterial DNA synthesis, and inhibition of cell division that leads to cell death. Moreover, AgNP releases Ag+ ion inside the bacterial cell [18]. Other study stated that possible mechanism involving the electrochemical proton gradient through respiratory processes in bacteria, which is the driving force for ATP synthesis. AgNPs may interrupt the energy source for all reactions that depend on energy, which leads to cell death because ATP synthesis enables cell adhesion and proliferation [17].

However, in this study, we found discoloration on the adhesive containing AgNP, a common side effect of silver-containing material. This is supported by the previous study stating that AgNP can cause grayish to blackish discoloration of material depending on the concentration used [16]. The result of this study can be a starting point for future studies regarding the manipulation of fixed retainer adhesive with antibacterial substance. It is clear that the addition of AgNPs has antibacterial effect. Furthermore, future research is needed for this formula to be used in human being.

\section{CONCLUSION}

AgNP-enhanced flowable composites in fixed retainers reduced T. denticola bacterial colony count. It could possibly inhibit periodontal disease as the side effect of fixed retainers which are commonly used for long term retention.

\section{ACKNOWLEDGMENT}

This research is approved and funded by HIBAH PITTA from Directorate Research and Community Engagement Universitas Indonesia.

\section{CONFLICTS OF INTEREST}

The authors report no conflicts of interest. 
Table 2: Treponema denticola colony count difference between groups with fixed retainer adhesive with and without AgNP

\begin{tabular}{|c|c|c|c|c|c|}
\hline Fixed retainer + Adhesive & $\mathbf{n}$ & Mean CFU/ $\mu \mathrm{L}$ & Median CFU/ $\mu \mathrm{L}$ & $\mathbf{t}$ & p value \\
\hline \multicolumn{6}{|l|}{ FRC } \\
\hline Without AgNP (F1) & 7 & 11.00 & $3 \times 10^{6}$ & \multirow[t]{2}{*}{-3.137} & \multirow[t]{2}{*}{$0.002^{* *}$} \\
\hline With AgNP (F2) & 7 & 4.00 & $6 \times 10^{4}$ & & \\
\hline \multicolumn{6}{|l|}{ MW } \\
\hline Without AgNP (M1) & 7 & 11.00 & $3 \times 10^{6}$ & \multirow[t]{2}{*}{-3.137} & \multirow[t]{2}{*}{$0.002^{* *}$} \\
\hline With AgNP (M2) & 7 & 4.00 & $13 \times 10^{4}$ & & \\
\hline \multicolumn{6}{|l|}{ Without AgNP } \\
\hline MW (M1) & 7 & 7.93 & $3 \times 10^{6}$ & -0.388 & 0.698 \\
\hline \multicolumn{6}{|l|}{ With AgNP } \\
\hline$\Delta \mathrm{FRC}$ & 7 & 7.86 & $6 \times 10^{4}$ & \multirow[t]{2}{*}{-0.320} & \multirow[t]{2}{*}{0.749} \\
\hline$\Delta \mathrm{MW}$ & 7 & 7.14 & $13 \times 10^{4}$ & & \\
\hline
\end{tabular}

${ }^{*} \mathrm{p}<0.05{ }^{* *} \mathrm{p}<0.01$. AgNP: Silver nanoparticle, CFU: Colony-forming unit, FRC: Fiber-reinforced composite, MW: Multistranded wires

Table 3: The difference between two types of fixed retainers for delta difference of Treponema denticola colony count (delta of with and without AgNP)

\begin{tabular}{llllll}
\hline Fixed retainer & $\mathbf{n}$ & Mean CFU/ $\boldsymbol{\mu L}$ & SD CFU/ $\boldsymbol{\mu L}$ & SE CFU/ $\boldsymbol{\mu L}$ & $\mathbf{p}$ value \\
\hline$\Delta \mathrm{FRC}$ & 7 & $2.8 \times 10^{6}$ & $1.3 \times 10^{6}$ & $0.5 \times 10^{6}$ & 0.276 \\
$\Delta \mathrm{MW}$ & 7 & $4.6 \times 10^{6}$ & $3.8 \times 10^{6}$ & $1.4 \times 10^{6}$ & \\
\hline
\end{tabular}

*p<0.05. AgNP: Silver nanoparticle, CFU: Colony-forming unit

FRC: Fiber-reinforced composite, MW: Multistranded wires, SD: Standard deviation

\section{REFERENCES}

1. Proffit WR. Contemporary Orthodontics. St. Louis: C.V. Mosby; 2013. p. 557-64.

2. Geserick M, Ball J, Wichelhaus A. Bonding fiber-reinforced lingual retainers with color-reactivating flowable composite. J Clin Orthod 2004;38:560-2.

3. Hegde N, Vinayak GR, Vinay P, Handa A. Bonded retainers in orthodontics : A review. Int J Dent Clin 2011;3:53-4.

4. Tacken MP, Cosyn J, De Wilde P, Aerts J, Govaerts E, Vannet BV, et al. Glass fibre reinforced versus multistranded bonded orthodontic retainers: A 2 year prospective multi-centre study. Eur J Orthod 2010;32:117-23.

5. Kučera J, Marek I. Unexpected complications associated with mandibular fixed retainers: A retrospective study. Am J Orthod Dentofacial Orthop 2016;149:202-11.

6. Morita Y, Imai S, Hanyuda A, Matin K, Hanada N, Nakamura Y, et al. Effect of silver ion coating of fixed orthodontic retainers on the growth of oral pathogenic bacteria. Dent Mater J 2014;33:268-74.

7. Pandis N, Vlahopoulos K, Madianos P, Eliades T. Long-term periodontal status of patients with mandibular lingual fixed retention. Eur J Orthod 2007;29:471-6.

8. Oldenburg SJ. Silver Nanoparticles : Properties and Applications Silver
Nanoparticles for Nanotoxicology Research. No. 730815; 2008. p. 2.

9. Wijnhoven SW, Peijnenburg WJ, Herberts CA, Hagens WI, Oomen AG, Heugens EH. Nano-silver: A review of available data and knowledge gaps in human and environmental risk assessment. Nanotoxicology 2009;3:109-38.

10. Tayab T, Shetty A, Kayalvizhi G. The clinical applications of fiber reinforced composites in all specialties of dentistry an overview. Int J Compos Mater 2015;5:18-24.

11. Rivera MF, Lee JY, Aneja M, Goswami V, Liu L, Velsko IM, et al. Polymicrobial infection with major periodontal pathogens induced periodontal disease and aortic atherosclerosis in hyperlipidemic apoE(null) mice. PLoS One 2013;8:e57178.

12. Dashper SG, Seers CA, Tan KH, Reynolds EC. Virulence factors of the oral spirochete Treponema denticola. J Dent Res 2011;90:691-703.

13. Gaibani P. Role of Treponema denticola in the Pathogenesis and Progression of Periodontal Disease. Dottorato Di Ricerca Oncologia e Patologia Sperimentale (Progetto n. 2 Patologia Sperimentale). Vol. 41. Italy: Università di Bologna; 2010. p. 120.

14. Chukkapalli SS, Rivera MF, Velsko IM, Lee JY, Chen H, Zheng D, et al. Invasion of oral and aortic tissues by oral spirochete Treponema denticola in apoE(-/-) mice causally links periodontal disease and atherosclerosis. Infect Immun 2014;82:1959-67.

15. Sela MN. Role of Treponema denticola in periodontal diseases. Crit Rev Oral Biol Med 2001;12:399-413.

16. Batra P, Miglani R. Antibacterial properties of retainers with silver nanoparticles. Am J Orthod Dentofac Orthop 2016;150:208-9.

17. Durán N, Durán M, de Jesus MB, Seabra AB, Fávaro WJ, Nakazato G, et al. Silver nanoparticles: A new view on mechanistic aspects on antimicrobial activity. Nanomedicine 2016;12:789-99.

18. Farhadian N, Usefi Mashoof R, Khanizadeh S, Ghaderi E, Farhadian M, Miresmaeili A, et al. Streptococcus mutans counts in patients wearing removable retainers with silver nanoparticles vs those wearing conventional retainers: A randomized clinical trial. Am J Orthod Dentofacial Orthop 2016;149:155-60. 